\title{
Waveform analysis
}

\author{
Accurate characterization of ultrafast optical pulses is important for applications such as spectroscopy \\ and communications research. S. J. Ben Yoo from the University of California at Davis explains his team's \\ scheme for real-time measurement of the amplitude and phase of arbitrary and non-repetitive waveforms.
}

\begin{abstract}
What is the motivation behind your work on waveform characterization?

The work in our lab focuses on investigating new techniques and devices for the generation and measurement of arbitrary optical waveforms. We have recently generated arbitrary optical waveforms by controlling the amplitude and phase of optical frequency combs on a line-by-line basis. The ultimate goal is to modulate the amplitude and phase, referred to as full-field modulation, of octave-spanning optical frequency combs. This will allow us to continuously generate optical waveforms with arbitrary temporal amplitude and phase profiles over long periods of time, ideally reaching timescales of many seconds. We must find a way of characterizing, in a single shot, the full field of broad-bandwidth arbitrary waveforms over long record times.
\end{abstract}

\section{What is your approach?}

We have demonstrated a technique that can achieve single-shot, dynamic, full-field, real-time and ultra-sensitive measurement of arbitrary optical waveforms, with a bandwidth that is scalable to the terahertz range and above. The scheme uses several important developments to overcome the limitations of previous techniques. First, to overcome electronic bandwidth limitations, we partitioned the signal spectrum into a number of spectral slices and processed them in parallel using an array of coherent homodyne receivers. Each spectral slice mixes with a single line from a stable optical frequency comb (a local oscillator) to generate the full-field time-domain information (in-phase and quadrature-phase components) at each coherent homodyne receiver. We then digitized the full-field signal from each spectral slice and synthesized it in real time across the entire spectrum. Central to the success of our approach is the chip-scale integration of the spectral demultiplexers and the $90^{\circ}$ optical hybrids. The wideband demultiplexer slices the optical signal into each spectral bin, and the narrowband demultiplexer splits the optical comb into each comb line. The optical hybrids coherently combine the comb line and the spectral slice of the signal in four quadrature

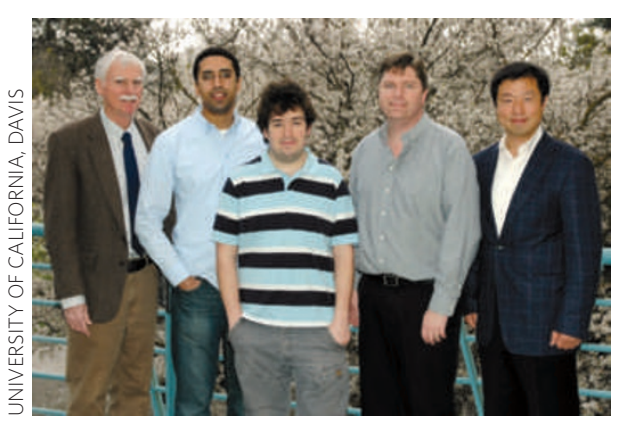

From left to right: Jonathan Heritage, Francisco Soares, Nicolas Fontaine, Ryan Scott and S. J. Ben Yoo. The team demonstrated a technique that can achieve single-shot, dynamic, full-field, real-time and ultrahigh-sensitive measurement of arbitrary optical waveforms with a scalable bandwidth.

phases at every demultiplexed channel. The integration of these devices assures optical coherence between the measured spectral slices over long periods of time, and allows reconstruction of the fullsignal field from the individual slices. We achieved single-shot capability and long record lengths by digitizing the signals in the time domain, scalability in spectral bandwidth and speed by exploiting parallel processing, and high sensitivity and fidelity measurements through coherent homodyne detection. We demonstrated single-shot, full-field measurement capability over an instantaneous bandwidth of more than $160 \mathrm{GHz}$ with a $2 \mu$ s record length, and illustrated the prospects for future scalability.

\section{What are the implications of your findings?}

We can now continuously measure the intensity and phase of arbitrary optical waveforms with bandwidths well beyond currently available high-speed electronics. The integration and parallelization mean that the technique can be scaled up to terahertz bandwidths and beyond. Another important implication of our results is that optical arbitrary waveforms may be generated through the reverse of this technique; that is, relatively narrow modulated spectral slices can be coherently combined to create broadband dynamic and continuous waveforms. Such a complete optical arbitrary waveform generation and measurement system, together with an octave-spanning comb, provides a unique instrument that has femtosecond temporal resolution and infinite spectral resolution, limited only by the record length.

\section{What are the potential applications?}

This generation and measurement scheme could provide a modulation-formatindependent transmitter/receiver architecture for use in ultrahigh-speed optical communications. The electronic compensation of optical impairments - both linear and possibly nonlinear in nature - is possible over large bandwidths at both the transmitter and receiver. The approach can also be used for secure communication that exploits code-hopping or encryption in amplitude and phase, coherent optical orthogonal frequency-division multiplexing communications, light detection and ranging systems or remote sensing, in which a wide spectral range and high resolution with quantum-limited detection sensitivity would be useful. Other potential applications include coherent control and monitoring of quantum systems and multicolour coherent femtosecond spectroscopy, in which different parts of the optical spectrum are simultaneously shaped and measured.

\section{What is the direction of future work?} We will pursue full and monolithic integration of the optics and electronics on a silicon CMOS-compatible platform to realize on-chip photonic and electronic processing with very high capacity, stability and manufacturability, as well as uniform performance. We will also extend the measurement capability over wider spectral bandwidths. We will try to achieve quantumlimited performance by using digitizers with high effective-number-of-bits to reduce electronic noise, and by integrating intelligent and adaptive digital signals processing.

\section{INTERVIEW BY RACHEL WON}

S. J. Ben Yoo and co-workers have an Article on real-time arbitrary optical waveform measurement on page 248 of this issue. 